\title{
Inhibition of FASN suppresses migration, invasion and growth in hepatoma carcinoma cells by deregulating the HIF-1 $\alpha /$ IGFBP1 pathway
}

\author{
JIAOJIAO GONG $^{1 *}$, SHASHA SHEN ${ }^{1 *}$, YIXUAN YANG ${ }^{3}$, SI QIN $^{1}$, LIFAN HUANG $^{1}$, HONGMIN ZHANG $^{1}$, \\ LING CHEN $^{1}$, YAQIN CHEN ${ }^{1}$, SHIYING LI ${ }^{1}$, SHA SHE $^{1}$, MIN YANG ${ }^{1}$, HONG REN $^{1}$ and HUAIDONG HU ${ }^{2}$ \\ ${ }^{1}$ Institute for Viral Hepatitis, Key Laboratory of Molecular Biology for Infectious Diseases \\ (Ministry of Education), Departments of ${ }^{2}$ Clinical Nutrition and ${ }^{3}$ Infectious Diseases, \\ The Second Affiliated Hospital of Chongqing Medical University, Chongqing 400010, P.R. China
}

Received November 13, 2016; Accepted January 23, 2017

DOI: $10.3892 /$ ijo.2017.3867

\begin{abstract}
Hepatocellular carcinoma is the second most common cause of cancer-related deaths worldwide. Due to a high propensity to metastasize, active angiogenesis and rapid proliferation, recurrence and poor prognosis are major obstacles for treatment and cure of this disease. However, the detailed mechanisms of how fatty acid synthase (FASN) promotes migration, invasion and healing in tumor cells remain unclear. In the present study, the previous results that FASN was expressed higher in cancer samples than in non-cancerous samples, and influenced migration, invasion of hepatoma carcinoma cells, were verified by immunohistochemistry, tissue microarrays, Transwell assay and wound healing assay. The secretory proteins of hepatocellular carcinoma cells with or without FASN knockdown were analyzed using the isobaric tags for relative and absolute quantitation (iTRAQ) method to identify differentially expressed proteins (DEPs). The DEPs were verified by RT-PCR and western blot analysis, and were consistent with the iTRAQ results. Inhibition of FASN can decrease the levels of IGFBP1, and the expression, activity, and ubiquitination of HIF-1 $\alpha$. Inhibition of FASN can suppress migration, invasion and healing of hepatoma carcinoma cells by decreasing HIF-1 $\alpha$, and IGFBP1.
\end{abstract}

\section{Introduction}

As the most common primary liver cancer, and the second most common cause of cancer-related deaths worldwide (1),

Correspondence to: Dr Huaidong Hu, Department of Clinical Nutrition, The Second Affiliated Hospital of Chongqing Medical University, 76 Linjiang Road, Chongqing 400010, P.R. China E-mail: huhuaidong@sina.com

"Contributed equally

Key words: hepatocellular carcinoma, fatty acid synthase, isobaric tags for relative and absolute quantitation, insulin-like growth factor binding protein $1, \mathrm{HIF}-1 \alpha$ hepatocellular carcinoma ( $\mathrm{HCC}$ ) is one of the most common cancers (2). In Asia, HCC is the second leading cause of cancer related deaths (3). Chronic infection with hepatitis $B$ and $C$, exposure to aflatoxin, smoking, excessive alcohol use and metabolic syndromes are the main etiological factors (4). Of these, HBV infection is the most common cause, especially in Asia (5). A high propensity to metastasize, active angiogenesis and rapid proliferation are the common causes of rapid recurrence and poor prognoses in HCC patients (6).

Fatty acid synthase (FASN) is a key enzyme for catalyzing endogenous fatty acid synthesis, a precursor of some biologically important lipids (7). Since the original determination that FASN is highly expressed in breast cancer and is related to tumor stage and prognosis, increasing number of studies have found that it has effect on tumor occurrence and development in various cancers, such as esophageal, lung, ovarian, prostate, nasopharyngeal and gastric cancers and malignant melanoma (8-11). As shown in a previous study, FASN contributed to migration and invasion in HCC cells (12) and osteosarcoma (OS) cells (13). However, few studies have focused on the mechanism by which FASN promotes migration and invasion of tumor cells.

In the present study, we took advantage of isobaric tags for relative and absolute quantitation (iTRAQ) method to identify the differentially expressed secretory proteins (DEPs) between HepG2215 cells with or without silencing of the FASN gene. After verification and further analysis by RT-PCR and western blot analysis, we identified a novel pathway which may explicate the promotional effect of FASN on metastasis of HCC.

\section{Materials and methods}

Reagents. The eight-plex iTraq kits were acquired from Applied Biosystems (Foster City, CA, USA). All electrophoresis reagents were purchased from Bio-Rad (Hercules, CA, USA). CytoSelect ${ }^{\mathrm{TM}}$ 24-well Cell Migration and Invasion assay kits (8 $\mu \mathrm{m}$, colorimetric format) were purchased from Cell Biolabs (San Diego, CA, USA). Opti-MEM was purchase from Gibco (San Diego, CA, USA). Lipofectamine 2000, FASN 
specific siRNA oligonucleotides (HSS103565, HSS176712, HSS176713) and a negative control (12935-400) were from Invitrogen (Carlsbad, CA, USA). Monoclonal antibodies against fatty acid synthase (FASN, ab128870), antithrombin-III (SERPINC1, ab126598), proprotein convertase subtilisin/kexin type 9 (PCSK9, ab181142), insulin-like growth factor binding protein 1 (IGFBP1, ab180948), hypoxia-inducible factor-1 $\alpha$ (HIF-1 $\alpha$, ab51608), ubiquitin (ab134953) were obtained from Abcam (Cambridge, MA, USA). Apolipoprotein E (APOE, TA805358), neutrophil gelatinase-associated lipocalin (LCN2, TA322583), translationally-controlled tumor protein (TPT1, TA314640) were from OriGene (Rockville, MD, USA). $\mathrm{CoCl}_{2}$ (S2619) was from Sigma (St. Louis, MO, USA), and MG132 (C8661) was purchased from Selleck Chemicals (Houston, TX, USA).

Cell lines. Human liver cancer cell lines, HepG2, HepG2215 and Bel7402, were acquired from ATCC and cultured in highglucose DMEM with $10 \%$ fetal bovine serum (FBS; Gibco), containing $1 \%$ penicillin and streptomycin. Cells were incubated in an atmosphere of $5.0 \% \mathrm{CO}_{2}$ at $37^{\circ} \mathrm{C}$.

Protein collection and iTRAQ labeling. Total secretory protein was collected, filtered by needle filter, and concentrated by centrifugation with an Amicon ${ }^{\circledR}$ centrifugal filter (Billerica, MA, USA). The protein concentration was determined using a 2-D Quant kit. For each sample, $100 \mu \mathrm{g}$ of protein was precipitated, denatured, cysteine blocked, and digested with sequencing-grade modified trypsin, according to the manufacturer's instructions. Labeling was performed as follows: pooled secretory protein samples without FASN knockdown were labeled with 117 and 118 tags; pooled secretory protein samples with FASN knockdown were labeled with 119 and 121 tags. Prior to analysis, the labeled samples were pooled.

Fractionation of peptides. Fractions of the iTRAQ-labeled peptides were solubilized with a Pharmalyte and urea (Amersham Biosciences) solution. After dissolution, the samples were applied to $\mathrm{pH}$ 3.0-10.0 IPG gel strips (Amersham Biosciences) for $14 \mathrm{~h}$ at $30 \mathrm{~V}$. The peptides were subsequently focused successively for $1 \mathrm{~h}$ at $500 \mathrm{~V}, 1 \mathrm{~h} 1,000 \mathrm{~V}$, and, finally, at $8,000 \mathrm{~V}$ for $8.5 \mathrm{~h}$ on IPG phor (Amersham Biosciences). Peptides were extracted from the gel by use of a solution containing $0.1 \%$ formic acid and $2 \%$ acetonitrile. The fractions were lyophilized in a vacuum concentrator and purified on a SPE C18 column. Prior to mass spectrometric analysis, the purified fractions were lyophilized and stored at $-20^{\circ} \mathrm{C}$.

Mass spectrometry. After purification, each iTRAQ-labeled sample was resuspended in a $2 \%$ acetonitrile solution containing $0.1 \%$ formic acid, and injected into a QStar ESI Quadrupole time-of-flight mass spectrometer (Applied Biosystems) coupled to online capillary LC system (Amsterdam, The Netherlands). A gradient series for each analysis was loaded on the C18-PepMap column (Dionex) to separate the peptide mixture at a flow rate of $0.3 \mu 1 / \mathrm{min}$. For data acquisition, positive ion mode was used with a $300-1,800 \mathrm{~m} / \mathrm{z}$ mass range. We selected the two most abundantly charged peptides $>20$ counts, and excluded peptides with a mass tolerance of $30 \mathrm{sec} \pm 50 \mathrm{mDa}$.
Data were processed using ProteinPilot v.2.0 (Applied Biosystems, Waltham, MA, USA) and searching in the Uniprotkb database. MMTS modification of cysteine was specified as a fixed modification. Protein identification was based on selection thresholds of ProtScore $>1.3$ or ProtScore $<0.77$, and false discovery rate P-values $<0.05$.

Immunohistochemistry (IHC) and tissue microarrays (TMA). Tissue microarrays containing $70 \mathrm{HCC}$ samples from different cases and 70 matched HCC adjacent normal tissue were obtained from US Biomax Inc. (Rockville, MD, USA). After being fixed with $1 \%$ formalin, the tissue samples were embedded in paraffin and cut into sections. The sections were then warmed in a $60^{\circ} \mathrm{C}$ oven, deparaffinized and rehydrated. After 3 washes with PBS (5 min per wash), the sections were subjected to heat-induced antigen retrieval in $0.01 \mathrm{M}$ citrate solution for $5 \mathrm{~min}$ in a microwave. Endogenous peroxidase activity was quenched by incubating the sections in $3 \% \mathrm{H}_{2} \mathrm{O}_{2}$ for $10 \mathrm{~min}$. The sections were blocked with bovine serum albumin (BSA) for $30 \mathrm{~min}$ and were incubated overnight with antibodies against FASN $(1: 200)$ at $4^{\circ} \mathrm{C}$. Detection was performed on an Envision/Horseradish Peroxidase system (Dako-Cytomation, Glostrup, Denmark), and all slides were counterstained with Gill's hematoxylin for evaluation under a light microscope.

For further analysis by tissue microarray, TMAs were baked at $60^{\circ} \mathrm{C}$, and immunohistochemical procedures were performed as above. The expression of FASN was assessed by semi-quantitative scoring of the staining intensity (scale 0-3) and the percentage of positive cells $(0-100 \%)$. The staining intensity and positive cell scores were then multiplied, generating a score ranging from 0 to 300 . In order to maintain the consistency of the measurements, the same qualified pathologist assessed each sample and interpreted the hematoxylin and eosin sections and analysis/scoring of IHC data.

FASN siRNA transfection, Transwell assay and wound healing assay. Cells were transfected with $50 \mathrm{nM}$ of FASN-specific siRNA or a negative control siRNA (12935-400) or blank control (Lipo2000) using Lipofectamine 2000 (Thermo Fisher Scientific, Carlsbad, CA, USA) and opti-MEM (Gibco). The cells were cultured in high-glucose DMEM with $10 \%$ fetal bovine serum after transfection. Wound healing and Transwell assays were conducted 2 days after transfection. When the cells cultured in 6-well plates reached $100 \%$ confluence, a sterile p200 pipette tip was used to incise a wound in the cell monolayer, and the debris was removed by gently washing with PBS. The resulting gap was examined at 0 and $24 \mathrm{~h}$ under a microscope. The capacity of cell migration was determined by the closure of the wound channel. The Transwell invasion was performed using a Cell Invasion assay kit (Cell Biolabs, Inc., Beijing, China), according to the manufacturer's protocol. As determined by trypan blue exclusion, $\sim 1 \times 10^{5}$ viable and siRNAtransfected cells were seeded in the upper chamber, separated from a lower chamber by an $8-\mu \mathrm{m}$ pore size membrane. ECM Matrix gel was used to determine the number of invading cells by CyQuant GR fluorescent dye $(560 \mathrm{~nm})$ at $24 \mathrm{~h}$ after seeding. The determination of FASN downregulation was made via western blot analysis. All experiments were performed in triplicate. 
Western blot analysis. Cells were lysed with lysis buffer, and protein concentration was determined via an Enhanced BCA Protein assay kit (Beyotime, Jiangsu, China). The proteins were separated by SDS-PAGE and subsequently transferred to PVDF membranes. The membranes were blocked with BSA for $1 \mathrm{~h}$ at room temperature, and incubated with the primary antibodies $(1: 500-1: 2,000)$ overnight at $4^{\circ} \mathrm{C}$. The membranes were washed three times with Tris-buffered saline containing Tween-20 (TBST) buffer and incubated with HRP-conjugated secondary antibodies at a dilution of 1:5,000. The membranes were washed again with TBST and visualized with an ECL detection system (Bio-Rad Laboratories). Western blot analyses were performed in triplicate.

RNA extraction and quantitative RT-PCR. Total RNA was extracted using TRIzol reagent (Invitrogen), according to the manufacturer's instructions. First-strand cDNA was synthesized from $2 \mu \mathrm{g}$ of total RNA, using a Reverse Transcription kit (Thermo Fisher Scientific, Waltham, MA, USA). A Fast PCR kit (KAPA SYBR, MA, USA) was used to perform RT-PCR with primers for SERPINC1 (Hs00379789_CE), APOE (Hs00185983_CE), LCN2 (Hs00761477_CE), PCSK9 (Hs00358728_CE), APOA1 (Hs00609868_CE), IGFBP1 (Hs00287487_CE), GAPDH (Hs00115502_CE), TPT1 (Hs00806865_CE), GDF15 (Hs00783664_CE).Quantification of gene expression was calculated according to the $2^{-\Delta \Delta C T}$ method. RT-PCR analyses were conducted in triplicate.

Detection of HIF-1 $\alpha$ activity. All reagents and equipment used in the Dual-Luciferase Reporter assay system were acquired from Promega Corp. (Madison, WI, USA). Detection of HIF-1 $\alpha$ activity was performed as described previously (14). The luciferase vector pGL3-HRE (encoding firefly luciferase) was used to monitor promoter activity and the pRL-SV40 plasmid (encoding Renilla luciferase) was used as normalization control. Each sample was set up with 3 parallel expriments.

HepG2215 cells were transiently transfected with FASN siRNA or control siRNA. After transfection, the cells were seeded into 24-well plates and cultured in DMEM supplemented with $10 \%$ fetal bovine serum in $5 \% \mathrm{CO}_{2}$ at $37^{\circ} \mathrm{C}$. After $24 \mathrm{~h}$, all cells were transiently transfected with $500 \mathrm{ng}$ pGL3HRE and $15 \mathrm{ng}$ pRL-SV40 constructs using Lipofectamine 2000. At $24 \mathrm{~h}$ post-transfection of plasmid, the promoter activities were determined via the Dual-luciferase assay system, and normalized against the internal control activity of Renilla luciferase. Each experiment was done in triplicate.

Transient transfection of pCMV-HIF-1 $\alpha$. The pCMV- HIF- $1 \alpha$ plasmid was acquired from The Institute for Viral Hepatitis, The Second Affiliated Hospital of Chongqing Medical University, Chongqing, China. Transfection was performed using Lipofectamine 2000, according to the manufacturer's instructions. Twenty-four hours before transfection, $5 \times 10^{5}$ HepG2212 cells were seeded into a 6-well plate and transfected with $1 \mu \mathrm{g}$ plasmid. Cells were treated with other conditions after cultured $48 \mathrm{~h}$ post transfection.

Immunoprecipitation (IP). Cells cultured under hypoxic conditions for $6 \mathrm{~h}$ were collected for immunoprecipitation. Briefly, the cells were lysed with IP lysis buffer (Beyotime) and the lysate was centrifuged to pellet the cellular debris. The supernatant was transferred to a separate microfuge tube, precleared with protein A/G agarose beads and centrifuged to pellet the beads and removed protein impurity. Again, the pellet was discarded and the supernatant was transferred to a sterile microfuge tube and incubated with mouse or rabbit IgG overnight at $4^{\circ} \mathrm{C}$. The following day, the supernatant was incubated with protein $\mathrm{A} / \mathrm{G}$ agarose beads coupled with the indicated antibodies for $>2 \mathrm{~h}$. The suspension was centrifuged to concentrate the beads, which then were washed thrice with IP lysis buffer ( 3 min per wash). The final pellet was resuspended with the $2 \mathrm{X}$ SDS loading buffer used for western blot analysis.

Statistical analysis. All experiments were conducted at least three times. Continuous variables are presented as the mean \pm standard deviation (SD). Comparisons between groups were analyzed by the Student's t-test or a Mann-Whitney U test. Qualitative variables are presented as counts and percentages, which were analyzed with the $\chi^{2}$ test. Differences in the data were considered to be statistically significant when the P-value was $<0.05$.

\section{Results}

Differential expression of FASN in tissues. To verify the previous determination that the expression of FASN was higher in HCC tissue, compared to normal tissue adjacent to HCC tissue, IHC was performed on tissue microarrays of hepatocellular carcinoma and non-cancerous tissues to identify the differences in FASN expression. The results showed that FASN was expressed higher in cancer samples than in non-cancerous samples (Fig. 1A). IHC score values of FASN were significantly higher in the HCC tissue group than in the HCC adjacent normal tissue group (Fig. 1B), similar to previous results.

Effect of FASN on cell migration and invasion in HCC. To validate the association of FASN to HCC migration and invasion, three FASN siRNA and control siRNA were used to transfect HepG2 and BEL7402 cell lines. The FASN siRNA significantly downregulated the expression of FASN (Fig. 2A and B). According to the results, it was found that the invasive ability of HepG2 and BEL7402 was significantly decreased after RNAi mediated silencing of FASN (Fig. 2D). According to the wound healing test, FASN knockdown resulted in a significant decrease in the migration ability of HepG2 and BEL7402 cells (Fig. 2C).

iTRAQ analysis of differentially expressed proteins. In order to study the mechanism by which FASN influences cell migration and invasion, iTRAQ-based MS was performed on proteins isolated from HepG2215 after siRNA-mediated FASN knockdown and from unsilenced control cells (Fig. 3). For ProteinPilot-based database searching and identification, the threshold was set to achieve $95 \%$ confidence at $5 \%$ false discovery rate. The iTRAQ results revealed that the protein profiles differed significantly between the FASN knockdown and unsilenced control samples. The ratios of 119:117 and 121:118 identified the differential protein expression and, 
A FASN

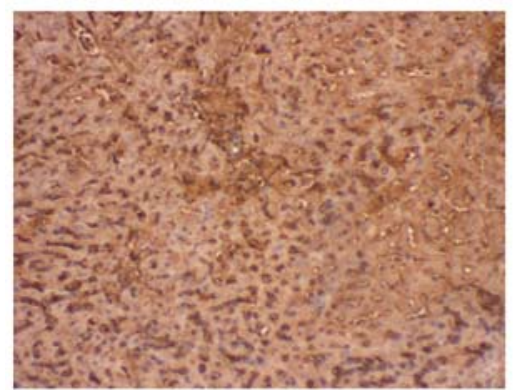

Tumor tissue

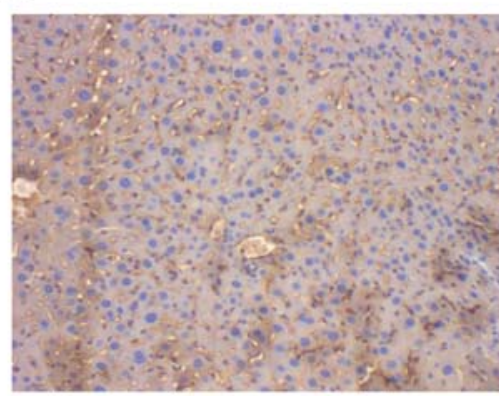

Tumor adjacent normal tissue

B

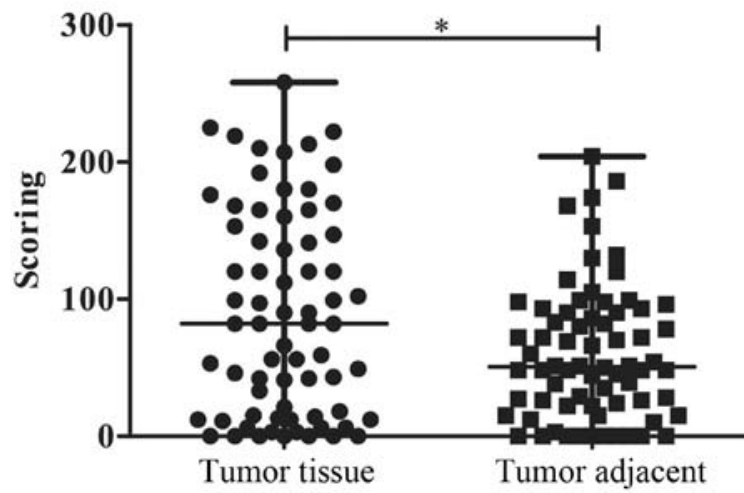

Figure 1. FASN levels were higher in HCC tissue than in adjacent normal tissue. (A) Representative images of the immmunohistochemical analysis of FASN in HCC tissue and matched HCC adjacent normal tissue. (B) IHC score values of FASN are significantly higher in HCC tissue compared to matched HCC adjacent normal tissue. ${ }^{*} \mathrm{P}<0.05$.
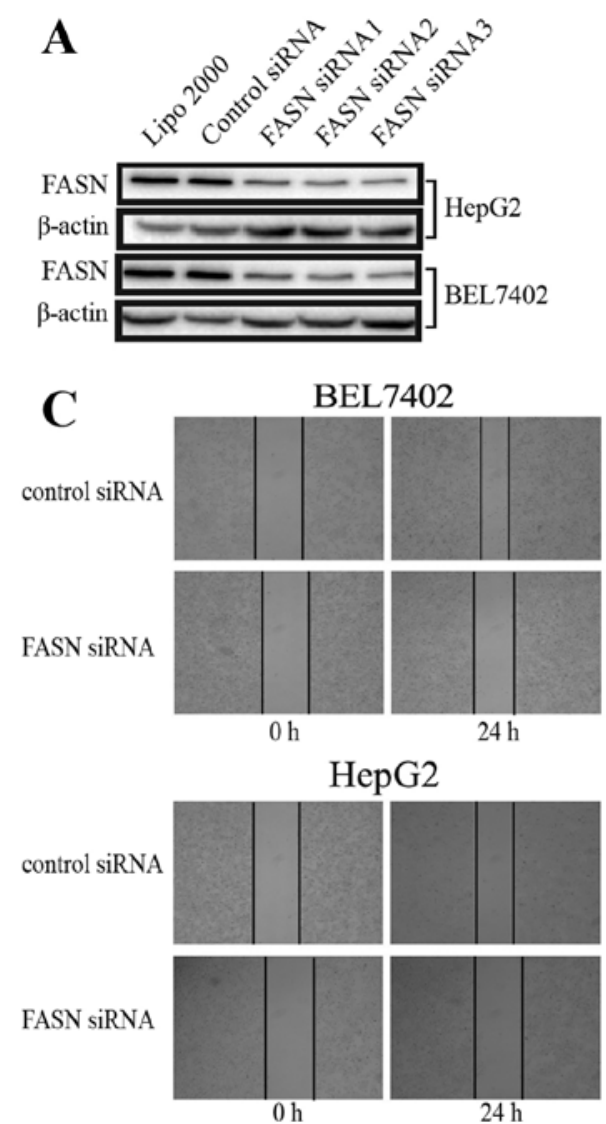
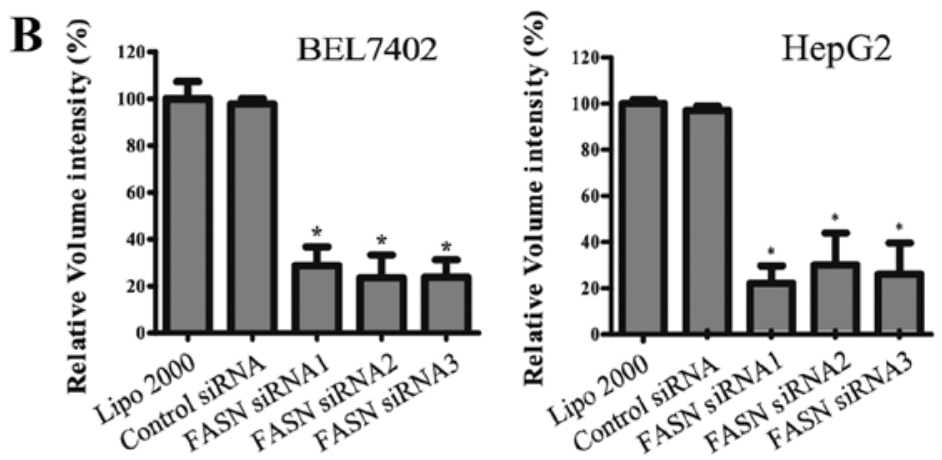

D

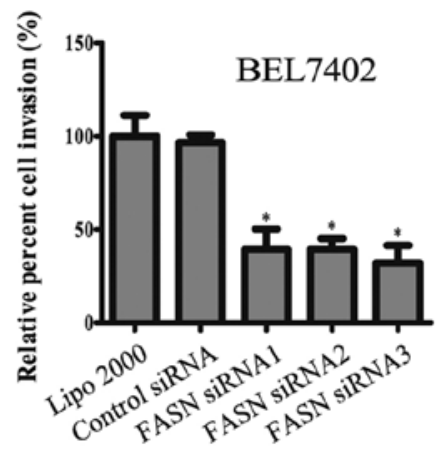

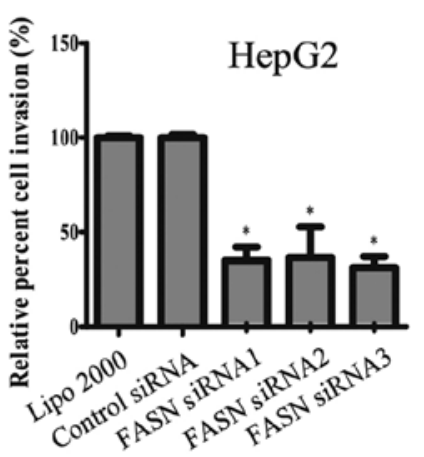

Figure 2. FASN plays a key role in cell migration and invasion in HCC. (A and B) Western blot analysis showed that three different siRNAs targeting FASNspecific siRNAs significantly reduced FASN protein levels in cell lysates, compared to control siRNA and lipo2000. (C) Migration ability of cells in wound healing assays after FASN knockdown. ${ }^{*} \mathrm{P}<0.05$. (D) Invasion ability of HepG2 and BEL7402 after silencing FASN. 


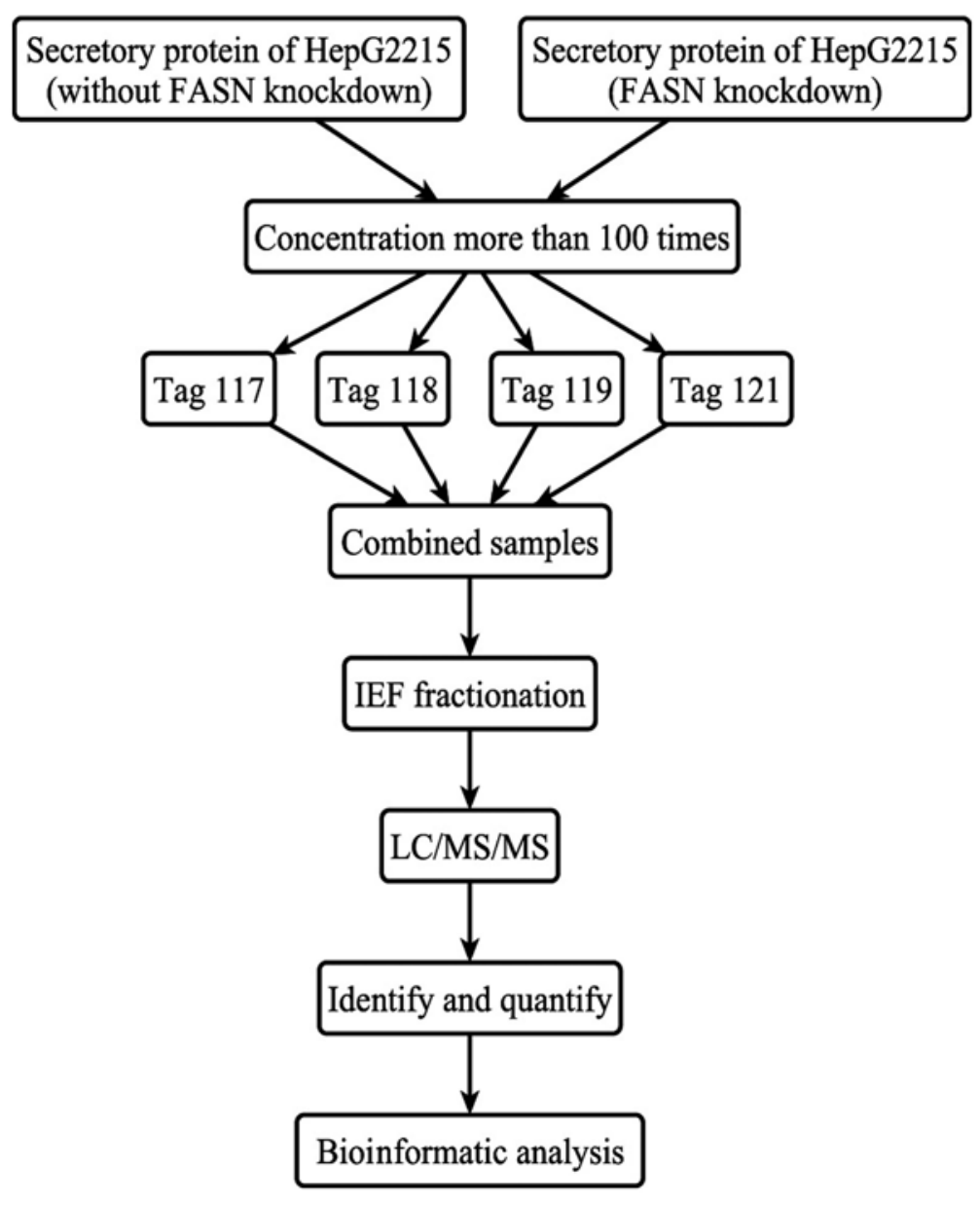

Figure 3. Flow chart of the iTRAQ-based MS proteomics approach used in this study.

according to the commonly adopted iTRAQ-based MS conventions (15), a ratio <0.77 (1/1.3) or >1.3 (1.3/1) was considered as significantly downregulated or upregulated, respectively. According to previous studies, technical variation $<30 \%$ can be achieved by repeat iTRAQ experiments.

A total of 30 proteins were identified with a confidence of $95 \%$, of which 8 were significantly upregulated and 22 downregulated (Table I). To identify the cellular and molecular characteristics of these proteins, the differentially expressed proteins were grouped according to their reported molecular functions and biological process using the PANTHER (www. pantherdb.org/) Classification System. The 30 DEPs hold 13 protein classes, 9 biological processes and 5 molecular functions (Fig. 4). The results showed that 'catalytic activity' and 'binding' were the most common molecular functions while 'metabolic process' was the most common biological process.

Validation of differentially expressed proteins. RT-PCR and western blot analyses were used to validate the differentially expressed proteins identified by iTRAQ. RT-PCR showed the mRNA expression levels of SERPINC1, APOA1, APOE, LCN2, PCSK9, GAPDH, IGFBP1, TPT1, GDF15, relative to $18 \mathrm{~S}$. The RT-PCR results were consistent with the results obtained via iTRAQ. After knockdown of FASN, the mRNA expression levels of SERPINC1, APOA1, APOE, LCN2, and
PCSK9 were significantly increased, while GAPDH, IGFBP1, TPT1 and GDF15 were decreased (Fig. 5A). Western blot analysis was utilized to quantify the expression levels of the identified proteins (Fig. 5B), and the results of the extracellular LCN2, SERPINC1, APOE, PCSK9, IGFBP1, TPT1, were consistent with the results achieved through iTRAQ analysis. The intracellular IGFBP1 and TPT1 also followed the above trend. However, the expression of intracellular IGFBP1 was significantly lower than extracellular levels. After silencing FASN, the intracellular APOE displayed no significant difference, and the intracellular levels of SERPINC1, LCN2, and PCSK9 were expressed so weakly that they could not be identified by western blot analysis.

FASN promotes the development of HCC by the effect of $H I F-1 \alpha$ to IGFBPI. Because IGFBP1 affects the growth, proliferation and metastasis of tumors (16), we investigated the expression of HIF- $1 \alpha$, which is one of the upstream precursors of IGFBP1. HIF-1 $\alpha$ activity was determined using the Dual-Luciferase Reporter assay system (Fig. 6). The results showed that the activity of HIF-1 $\alpha$ in the FASN-silenced group was decreased significantly compared to the control group $(\mathrm{P}=0.0008)$. To determine whether FASN regulated IGFBP1 by influencing HIF-1 $\alpha$, we conducted western blot analyses to monitor the expression of HIF-1 $\alpha$ under FASN knockdown conditions (Fig. 7A and B). In addition, western blot analyses 


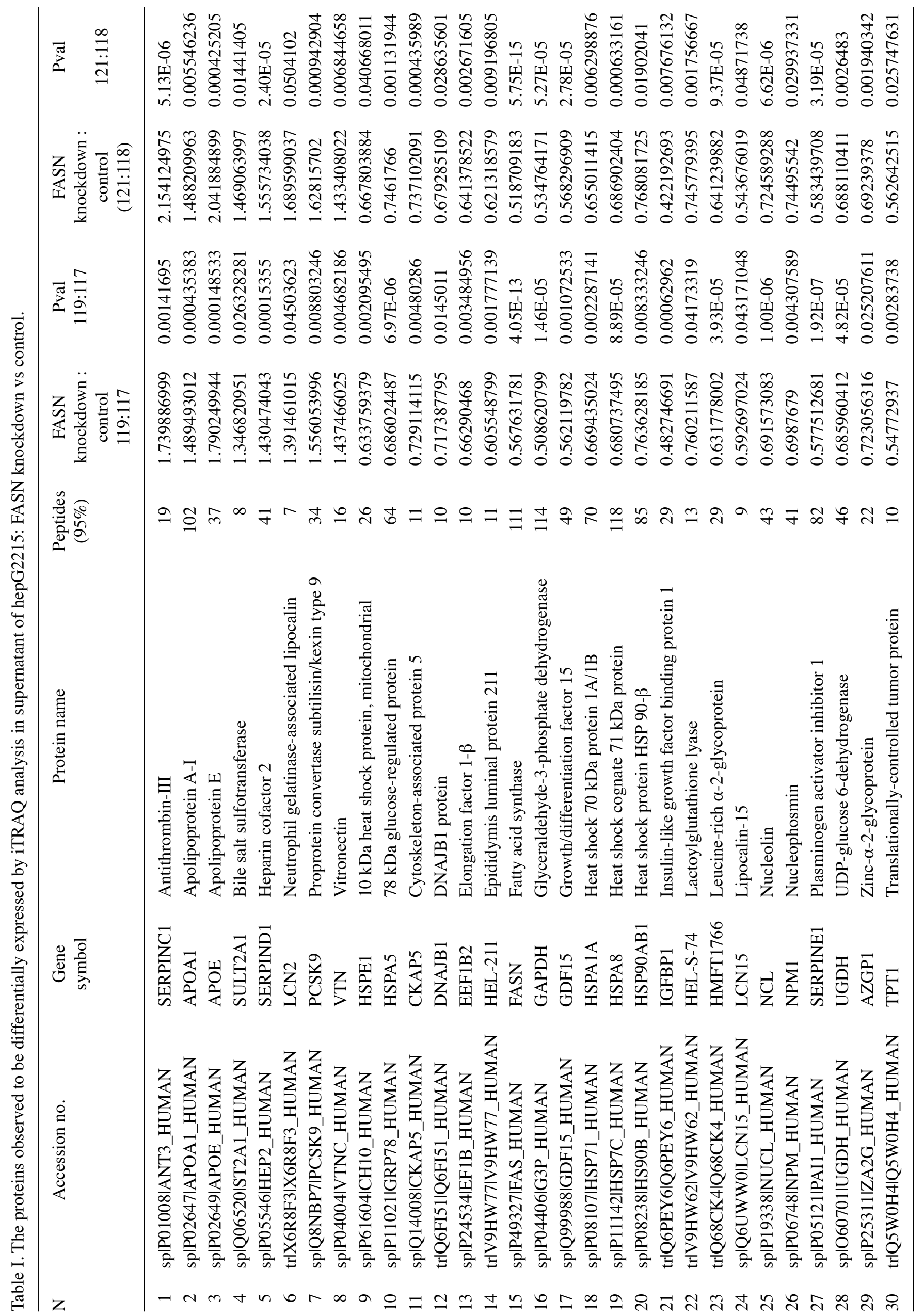




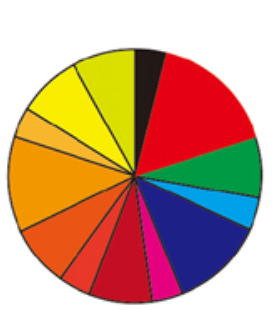

- cell adhesion molecule (PC00069) $4.0 \%$

chaperone (PC00072) $16.0 \%$

cytoskeletal protein (PC00085) $8.0 \%$

$\square$ defense/immunity protein (PC00090) 4.0\%

- enzyme modulator (PC00095) $12.0 \%$

hydrolase (PC00121) 4.0\%

- isomerase (PC00135) 8.0\%

$\square$ ligase (PC00142) 4.0\%

$\square$ nucleic acid binding (PC00171) 8.0\%

$\square$ oxidoreductase (PC00176) 12.0\%

$\square$ signaling molecule (PC00207) 4.0\%

$\square$ transfer/carrier protein (PC00219) $8.0 \%$

PANTHER Protein Class $\square$ transferase (PC00220) $8.0 \%$

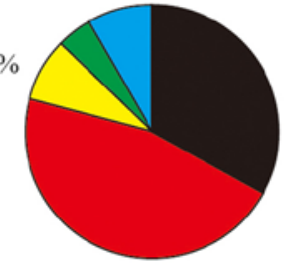

GO Molecular Function

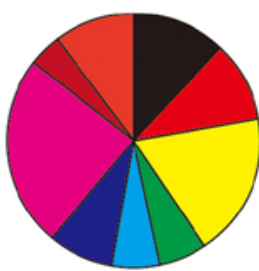

a biological regulation (GO:0065007) $12.2 \%$

cellular component organization or biogenesis (GO:0071840) $10.2 \%$

$\square$ cellular process (GO:0009987) 18.4\%

$\square$ developmental process (GO:0032502) $6.1 \%$

$\square$ immune system process (GO:0002376) $6.1 \%$

localization (GO:0051179) $8.2 \%$

metabolic process (GO:0008152) $24.5 \%$

multicellular organismal process (GO:0032501) $4.1 \%$

$\square$ response to stimulus (GO:0050896) $10.2 \%$

GO Biological Process

Figure 4. PANTHER analysis of proteins aberrantly expressed in HCC cells. Proteins were categorized by protein class, biological process, and molecular function.

A

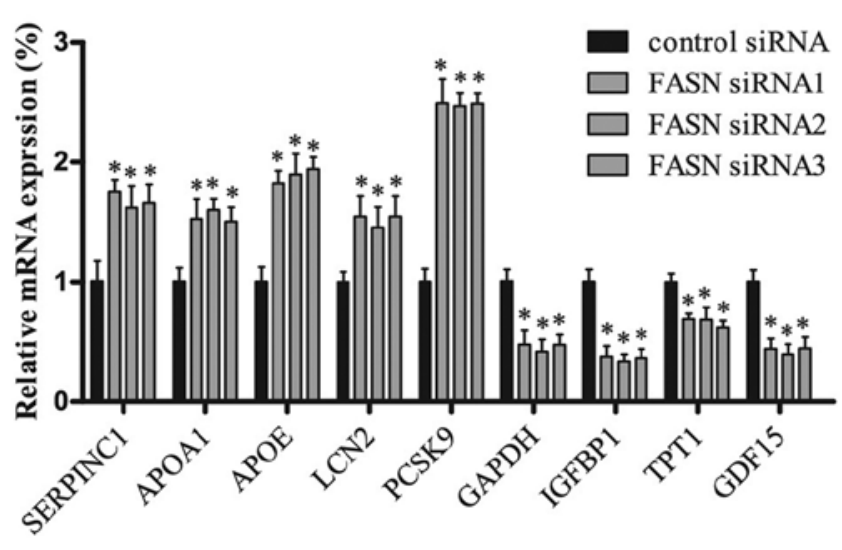

B

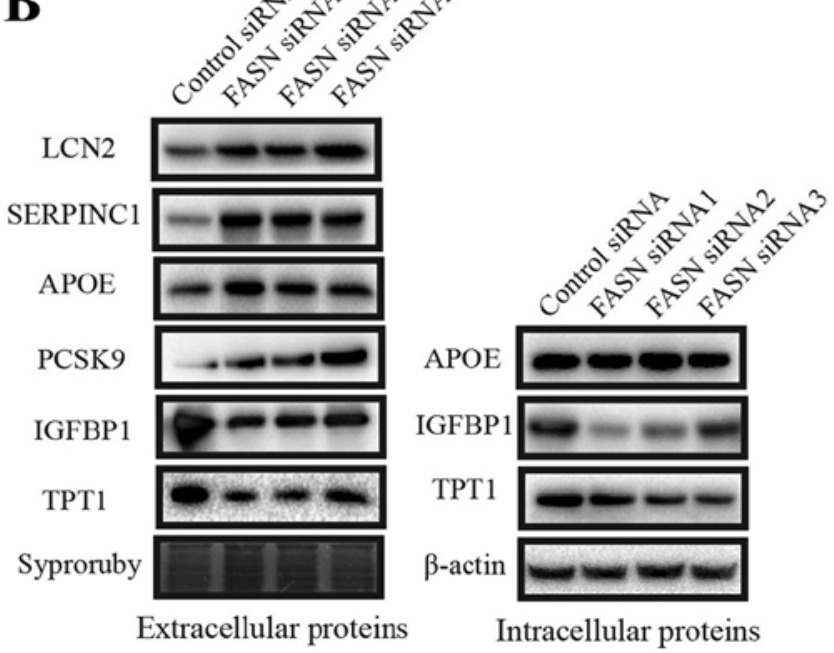

Figure 5. Validation of differentially expressed proteins. (A) RT-PCR detected the relative mRNA expression levels of some DEPs in the FASN knockdown group compared with untreated (no knockdown) group. (B) A representative western blot analysis for SERPINC1, APOE, LCN2, PCSK9, IGFBP1, TPT1 expression in the experimental and control groups. ${ }^{*} \mathrm{P}<0.05$.

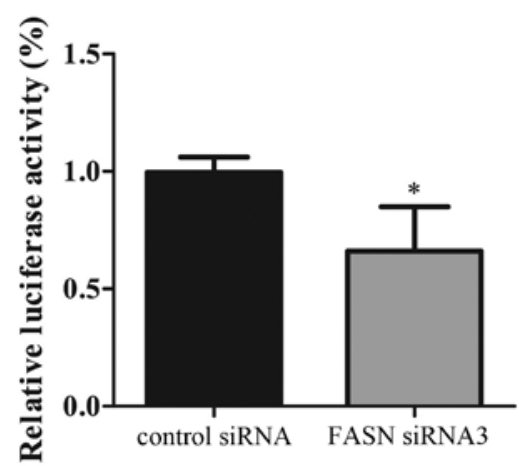

Figure 6. Inhibition of FASN downregulates the transcriptional activity of HIF-1 $\alpha$ in cobalt chloride-induced hypoxic conditions. HepG2215 cells exposed to hypoxia for $6 \mathrm{~h}$. ${ }^{*} \mathrm{P}<0.05$. monitored the expression of FASN when HIF-1 $\alpha$ overexpression was induced by pCMV-HIF-1 $\alpha$ (Fig. 7C), and these results were compared to control siRNA or plasmid control conditions. The results suggested that FASN may regulate HIF- $1 \alpha$ and therefore influence IGFBP1. This pathway might be the mechanism by which FASN affects proliferation and metastasis of HCC.

FASN affects HIF-la ubiquitination. It is known that many pathways regulate HIF-1 $\alpha$ (17), but the degradation pathway through ubiquitination-mediated proteasome is the most important regulator for cellular HIF-1 $\alpha$ levels (18). We identified the influence of FASN for HIF-1 $\alpha$ ubiquitination by western blot analysis after immunoprecipitation (Fig. 8). After 


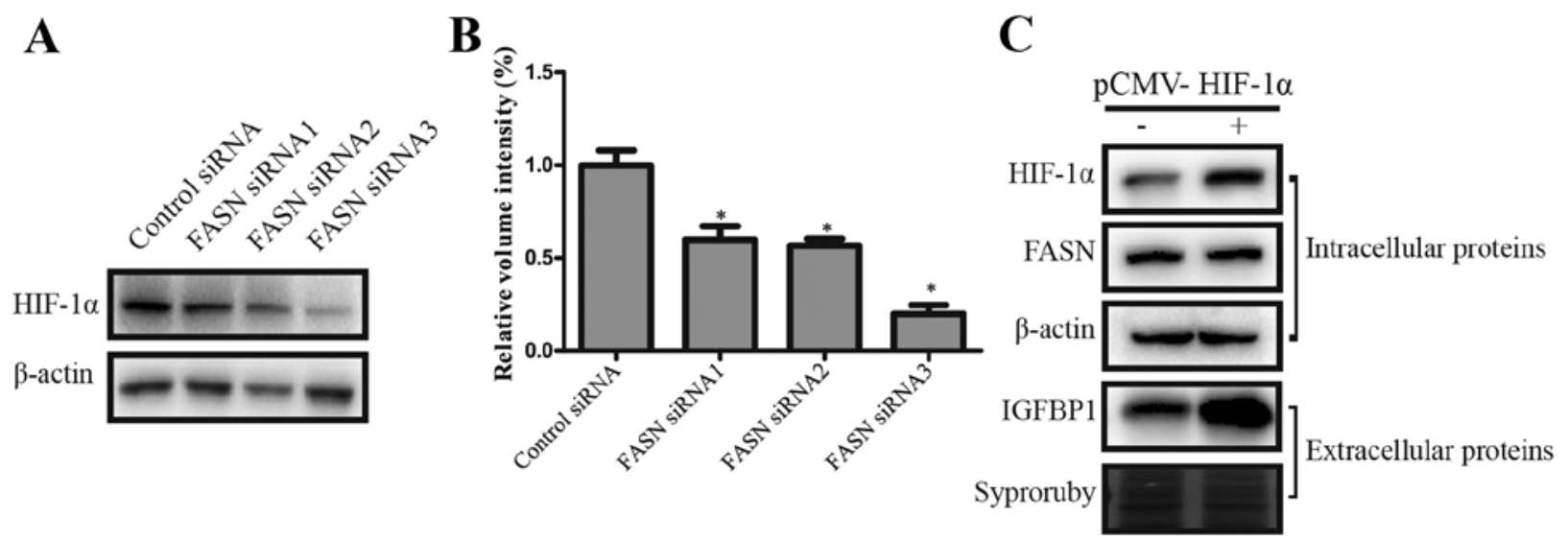

Figure 7. Effect of FASN on IGFBP1 was based on HIF-1 $\alpha$ in HepG2215. (A and B) Western blot analysis showed the expression levels of HIF-1 $\alpha$ in cells treated with three different siRNAs targeting FASN compared to control siRNA. (C) Western blot analysis showed the level of FASN and IGFBP1 when HIF-1 $\alpha$ overexpression was induced by pCMV-HIF-1 $\alpha$ plasmid. ${ }^{\mathrm{P}}<0.05$.

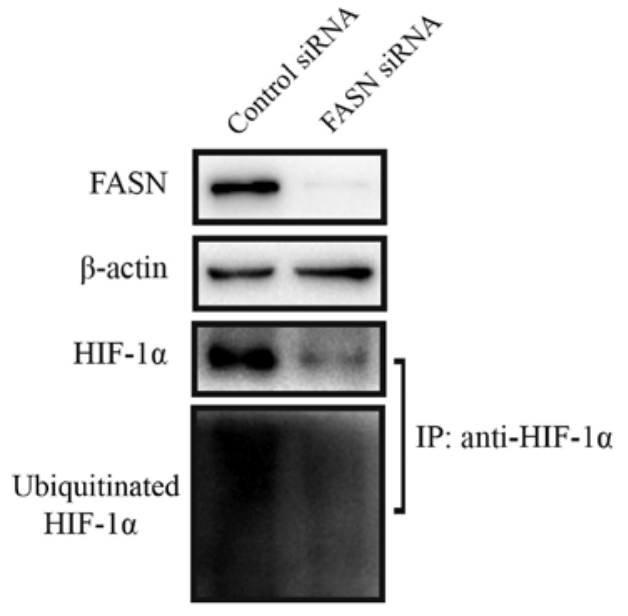

Figure 8. FASN affects HIF-1 $\alpha$ ubiquitination. HepG2215 cells induced to mimic hypoxic conditions for $6 \mathrm{~h}$. The proteasome inhibitor MG132 was used to block HIF-1 $\alpha$ degradation.

FASN knockdown, the HepG2215 cells were treated with $\mathrm{CoCl}_{2}(100 \mu \mathrm{M})$, to induce a hypoxic response, and MG132 $(10 \mu \mathrm{M})$, to inhibit proteasome directed HIF-1 $\alpha$ degradation. The results revealed that inhibiting FASN could downregulate HIF-1 $\alpha$ ubiquitination.

\section{Discussion}

The incidence of HCC is increasing continuously, and diagnosis is frequently made only during the late stages of disease (19). Although surgical resection and liver transplantation are the most successful treatments (20), patients suffering from advanced stage HCC have a high mortality rate. Metastasis and recurrence are the major obstacles to improvement of survival rates and quality of life in HCC patients (21). In recent years, many studies have confirmed that the occurrence of tumors is closely related to abnormal cell metabolism (22). FASN, as the key molecule for catalyzing fatty acid synthesis, is highly expressed in a number of malignant tumors. In this study, the results of the comparison of FASN expression between liver tumor tissue and adjacent non-cancerous tissue were in accordance with previous reports (23). We further found that FASN could significantly inhibit cell migration and invasion in Transwell assays and in the wound healing test.

According to the iTRAQ results, the majority of the DEPs were involved in metabolic and cellular processes. We identified many of them, including SERPINC1, APOA1, APOE, LCN2, PCSK9, GAPDH, IGFBP1, TPT1 and GDF15 using RT-PCR. Some of them were identified by western blot analysis. According to the results, the iTRAQ labeling method is both reliable and powerful for protein quantification. Based on protein classes, biological processes and molecular functions, we further classified the DEPs into different types, and noted the major changes after silencing FASN.

IGFBP1 belongs to the insulin-like growth factor binding protein gene family, which consists of six well-characterized members that encode a family of homologous multifunctional proteins, IGFBP-1 to IGFBP-6. However, in contrast to other proteins of this family, the expression of IGFBP-1 is markedly altered by changes in metabolic state (24). Jones et al have reported that IGFBP1-stimulated $\mathrm{CHO}$ cell migration is mediated by the $\alpha_{5} \beta_{1}$ integrin, but is independent of IGF-I (25). Several studies have reported that HIF-1 $\alpha$-responsive genes in mammals include the IGFBP-1 (26-28). HIF-1, consisting of HIF-1 $\alpha$ and HIF-1 $\beta$, and HIF-2 play a pivotal role in cellular adaptation to hypoxia and regulate the expression of genes responsible for glucose metabolism, angiogenesis, and cell survival (29). Based on the close relationship between HIF-1 $\alpha$ and IGFBP-1, and the observation that IGFBP-1 was significantly decreased when FASN was silenced, we explored further to identify the mechanism by which silencing FASN reduced expression of HIF- $1 \alpha$.

Studies have reported that FASN inhibition regulates the expression of HIF-1 $\alpha$ to various degrees in cancer cells (30-32). However, few studies have reported on the relationship of FASN and HIF-1 $\alpha$ in HCC. Our results revealed that the level of FASN expression can influence the level of HIF-1 $\alpha$, but HIF- $1 \alpha$ did not significantly regulate the expression of FASN. Additionally, measurements of HIF- $1 \alpha$ activity, after knockdown of FASN, showed that FASN promoted HIF-1 $\alpha$ transcriptional activity. When silencing FASN, the decrease in IGFBP1 may be through this pathway. 
The ubiquitin-proteasome is an important pathway for protein degradation, and is the major regulator for HIF-1 $\alpha \mathrm{s}$ in cells. After treating cells with $\mathrm{CoCl}_{2}$, to mimic a hypoxic environment, and MG132, to block HIF-1a degradation, the level of HIF-1 $\alpha$ and ubiquitination were significantly decreased in the FASN knockdown group, compared to the control group. The results indicated that FASN has an effect on ubiquitination of HIF-1 $\alpha$.

Lu et al considered that if FASN is a target gene of HIF, FASN levels would change with the change of HIF levels (33). However, Zhou et al reported inhibition of FASN blocked HIF-1 $\alpha$ in glioma cells (32). In different tissues, the relationship of FASN and HIF-1 $\alpha$ is different. In the present study, we determined that inhibition of FASN decreased the level of HIF-1 $\alpha$, IGFBP1, HIF-1 $\alpha$ activity and ubiquitination, in the HepG2215 cell line. As is known, FASN and HIF-1 $\alpha$ could promoted the occurrence, development and metastasis of tumors $(13,23,33-39)$. Especially for the promotional effect of HIF-1 $\alpha$ on tumor cell metastasis, many previous studies have indicated this conclusion, and reported some relevant pathways $(14,40-43)$. It is reasonable, then, to conclude that the effect of FASN on the proliferation, migration, invasion, and metastasis of hepatoma carcinoma cells, and angiogenesis within tumor tissues, is based on the role of HIF-1 $\alpha$. In future, these findings can bring some new insights for the treatment of HCC. FASN may be a new target for inhibiting tumor development and metastasis, and for improving the survival rate and quality of life of patients with hepatocellular carcinoma.

In conclusion, the present study provides a novel mechanism by which FASN promotes the proliferation, migration, invasion, metastasis of hepatocellular carcinoma cells, and angiogenesis in tumor tissues. Inhibition of FASN suppresses migration, invasion and healing of hepatoma carcinoma cells by decreasing HIF-1 $\alpha$ activity and IGFBP1.

\section{Acknowledgements}

This study was supported by the National Natural Science Foundation of China (81171560), National Key Technology Support Program (2012BAI35B03), the 'Par-Eu Scholars Program' of Chongqing City, and the National Science and Technology Major Project of China (2012ZX10002007001).

\section{References}

1. Torre LA, Bray F, Siegel RL, Ferlay J, Lortet-Tieulent J and Jemal A Global cancer statistics, 2012. CA Cancer J Clin 65: 87-108, 2015.

2. Ferlay J, Shin HR, Bray F, Forman D, Mathers C and Parkin DM: Estimates of worldwide burden of cancer in 2008: GLOBOCAN 2008. Int J Cancer 127: 2893-2917, 2010.

3. Jemal A, Bray F, Center MM, Ferlay J, Ward E and Forman D: Global cancer statistics. CA Cancer J Clin 61: 69-90, 2011.

4. Colombo M and Sangiovanni A: Hepatocellular carcinoma. Recenti Prog Med 107: 386-394, 2016 (In Italian).

5. Dondeti MF, El-Maadawy EA and Talaat RM: Hepatitis-related hepatocellular carcinoma: Insights into cytokine gene polymorphisms. World J Gastroenterol 22: 6800-6816, 2016.

6. Sucandy I, Cheek S, Golas BJ, Tsung A, Geller DA and Marsh JW: Longterm survival outcomes of patients undergoing treatment with radiofrequency ablation for hepatocellular carcinoma and metastatic colorectal cancer liver tumors. HPB Oxf 18: 756-763, 2016.

7. Liu H, Liu JY, Wu X and Zhang JT: Biochemistry, molecular biology, and pharmacology of fatty acid synthase, an emerging therapeutic target and diagnosis/prognosis marker. Int J Biochem Mol Biol 1: 69-89, 2010.
8. Cerne D, Zitnik IP and Sok M: Increased fatty acid synthase activity in non-small cell lung cancer tissue is a weaker predictor of shorter patient survival than increased lipoprotein lipase activity. Arch Med Res 41: 405-409, 2010.

9. Daker M, Bhuvanendran S, Ahmad M, Takada K and Khoo AS: Deregulation of lipid metabolism pathway genes in nasopharyngeal carcinoma cells. Mol Med Rep 7: 731-741, 2013.

10. Grunt TW, Wagner R, Grusch M, Berger W, Singer CF, Marian B Zielinski CC and Lupu R: Interaction between fatty acid synthase- and ErbB-systems in ovarian cancer cells. Biochem Biophys Res Commun 385: 454-459, 2009.

11. Zhou Y, Niu C, Li Y, Gao B, Zheng J, Guo X and Ma W: Fatty acid synthase expression and esophageal cancer. Mol Biol Rep 39: 9733-9739, 2012

12. Hao Q, Li T, Zhang X, Gao P, Qiao P, Li S and Geng Z: Expression and roles of fatty acid synthase in hepatocellular carcinoma. Oncol Rep 32: 2471-2476, 2014.

13. Long XH, Mao JH, Peng AF, Zhou Y, Huang SH and Liu ZL: Tumor suppressive microRNA-424 inhibits osteosarcoma cell migration and invasion via targeting fatty acid synthase. Exp Ther Med 5: 1048-1052, 2013.

14. Shi T, Dong Y, Li J, Gao P, Fu D and Ma D: High-throughput screening identifies CHMP4A associated with hypoxia-inducible factor 1. Life Sci 87: 604-608, 2010.

15. Gan CS, Chong PK, Pham TK and Wright PC: Technical, experimental, and biological variations in isobaric tags for relative and absolute quantitation (iTRAQ). J Proteome Res 6: 821-827, 2007.

16. Firth SM and Baxter RC: Cellular actions of the insulin-like growth factor binding proteins. Endocr Rev 23: 824-854, 2002.

17. Frede S, Berchner-Pfannschmidt U and Fandrey J: Regulation of hypoxia-inducible factors during inflammation. Methods Enzymol 435: 405-419, 2007.

18. Richard DE, Berra E, Gothié E, Roux D and Pouysségur J: p42/p44 mitogen-activated protein kinases phosphorylate hypoxia-inducible factor 1alpha (HIF-1alpha) and enhance the transcriptional activity of HIF-1. J Biol Chem 274: 32631-32637, 1999.

19. Qiao M, Sheng S and Pardee AB: Metastasis and AKT activation. Cell Cycle 7: 2991-2996, 2008.

20. Belghiti J and Fuks D: Liver resection and transplantation in hepatocellular carcinoma. Liver Cancer 1: 71-82, 2012.

21. Schwartz M, Roayaie S and Llovet J: How should patients with hepatocellular carcinoma recurrence after liver transplantation be treated? J Hepatol 43: 584-589, 2005.

22. Cairns RA, Harris I, McCracken S and Mak TW: Cancer cell metabolism. Cold Spring Harb Symp Quant Biol 76: 299-311, 2011.

23. Zhang H,Li H, Yang Y,Li S, Ren H,Zhang D and Hu H: Differential regulation of host genes including hepatic fatty acid synthase in HBV-transgenic mice. J Proteome Res 12: 2967-2979, 2013.

24. Lee PD, Giudice LC, Conover CA and Powell DR: Insulin-like growth factor binding protein-1: Recent findings and new directions. Proc Soc Exp Biol Med 216: 319-357, 1997.

25. Jones JI, Gockerman A, Busby WH Jr, Wright G and Clemmons DR: Insulin-like growth factor binding protein 1 stimulates cell migration and binds to the alpha 5 beta 1 integrin by means of its Arg-Gly-Asp sequence. Proc Natl Acad Sci USA 90: 10553-10557, 1993.

26. Semenza GL: Oxygen sensing, homeostasis, and disease. N Engl J Med 365: 537-547, 2011.

27. Sugawara J, Tazuke SI, Suen LF, Powell DR, Kaper F, Giaccia AJ and Giudice LC: Regulation of insulin-like growth factor-binding protein 1 by hypoxia and 3',5'-cyclic adenosine monophosphate is additive in HepG2 cells. J Clin Endocrinol Metab 85: 3821-3827, 2000.

28. Tuomisto TT, Rissanen TT, Vajanto I, Korkeela A, Rutanen J and Ylä-Herttuala S: HIF-VEGF-VEGFR-2, TNF-alpha and IGF pathways are upregulated in critical human skeletal muscle ischemia as studied with DNA array. Atherosclerosis 174: 111-120, 2004.

29. Bertout JA, Patel SA and Simon MC: The impact of O2 availability on human cancer. Nat Rev Cancer 8: 967-975, 2008.

30. Menendez JA, Vellon L, Oza BP and Lupu R: Does endogenous fatty acid metabolism allow cancer cells to sense hypoxia and mediate hypoxic vasodilatation? Characterization of a novel molecular connection between fatty acid synthase (FAS) and hypoxia-inducible factor-1alpha (HIF-lalpha)-related expression of vascular endothelial growth factor (VEGF) in cancer cells overexpressing her-2/neu oncogene. J Cell Biochem 94: 857-863, 2005. 
31. Seguin F, Carvalho MA, Bastos DC, Agostini M, Zecchin KG Alvarez-Flores MP, Chudzinski-Tavassi AM, Coletta RD and Graner E: The fatty acid synthase inhibitor orlistat reduces experimental metastases and angiogenesis in B16-F10 melanomas. $\mathrm{Br}$ J Cancer 107: 977-987, 2012.

32. Zhou Y, Jin G, Mi R, Zhang J, Zhang J, Xu H, Cheng S, Zhang Y, Song W and Liu F: Inhibition of fatty acid synthase suppresses neovascularization via regulating the expression of VEGF-A in glioma. J Cancer Res Clin Oncol 142: 2447-2459, 2016.

33. Lu GY, Huang SM, Liu ST, Liu PY, Chou WY and Lin WS: Caffeine induces tumor cytotoxicity via the regulation of alternative splicing in subsets of cancer-associated genes. Int J Biochem Cell Biol 47: 83-92, 2014.

34. Chang L, Wu P, Senthilkumar R, Tian X, Liu H, Shen X, Tao Z and Huang P: Loss of fatty acid synthase suppresses the malignant phenotype of colorectal cancer cells by down-regulating energy metabolism and mTOR signaling pathway. J Cancer Res Clin Oncol 142: 59-72, 2016.

35. Liu ZL, Mao JH, Peng AF, Yin QS, Zhou Y, Long XH and Huang SH: Inhibition of fatty acid synthase suppresses osteosarcoma cell invasion and migration via downregulation of the PI3K/Akt signaling pathway in vitro. Mol Med Rep 7: 608-612, 2013.

36. Mao JH, Zhou RP, Peng AF, Liu ZL, Huang SH, Long XH and Shu Y: microRNA-195 suppresses osteosarcoma cell invasion and migration in vitro by targeting FASN. Oncol Lett 4: 1125-1129, 2012.

37. Singh R, Yadav V, Kumar S and Saini N: MicroRNA-195 inhibits proliferation, invasion and metastasis in breast cancer cells by targeting FASN, HMGCR, ACACA and CYP27B1. Sci Rep 5: 17454,2015
38. Wang TF, Wang H, Peng AF, Luo QF, Liu ZL, Zhou RP, Gao S, Zhou Y and Chen WZ: Inhibition of fatty acid synthase suppresses U-2 OS cell invasion and migration via downregulating the activity of HER2/PI3K/AKT signaling pathway in vitro. Biochem Biophys Res Commun 440: 229-234, 2013.

39. Yu B, Miao ZH, Jiang Y, Li MH, Yang N, Li T and Ding J: c-Jun protects hypoxia-inducible factor-1alpha from degradation via its oxygen-dependent degradation domain in a nontranscriptional manner. Cancer Res 69: 7704-7712, 2009.

40. Cai Q, Wang Z, Wang S, Weng M, Zhou D, Li C, Wang J, Chen E and Quan Z: Long non-coding RNA LINC00152 promotes gallbladder cancer metastasis and epithelial-mesenchymal transition by regulating HIF-1alpha via miR-138. Open Biol 7: 160247, 2017.

41. Maroni P, Matteucci E, Bendinelli P and Desiderio MA: Functions and epigenetic regulation of Wwox in bone metastasis from breast carcinoma: Comparison with primary tumors. Int J Mol Sci 18: 18, 2017.

42. Sun LL, Song Z, Li WZ and Tang SY: Hypoxia facilitates epithelial-mesenchymal transition-mediated rectal cancer progress. Genet Mol Res 15: 15, 2016.

43. Yan X, Jiao SC, Zhang GQ, Guan Y and Wang JL: Tumorassociated immune factors are associated with recurrence and metastasis in non-small cell lung cancer. Cancer Gene Ther: doi: 10.1038/cgt.2016.40 2017. 